This item was submitted to Loughborough's Research Repository by the author.

Items in Figshare are protected by copyright, with all rights reserved, unless otherwise indicated.

\title{
Reachability analysis of landing sites for forced landing of a UAS in wind using trochoidal turn paths
}

PLEASE CITE THE PUBLISHED VERSION

https://doi.org/10.1109/ICUAS.2015.7152276

PUBLISHER

IEEE

VERSION

SMUR (Submitted Manuscript Under Review)

LICENCE

CC BY-NC-ND 4.0

\section{REPOSITORY RECORD}

Coombes, Matthew, Wen-Hua Chen, and Peter M. Render. 2015. "Reachability Analysis of Landing Sites for Forced Landing of a UAS in Wind Using Trochoidal Turn Paths". figshare. https://hdl.handle.net/2134/18006. 


\title{
Reachability Analysis of Landing Sites for Forced Landing of a UAS in Wind using Trochoidal Turn Paths
}

\author{
Matthew Coombes, Wen-Hua Chen, Peter Render \\ Department of Automotive and Aeronautical Engineering \\ Loughborough University, Loughborough, LE11 3TQ UK \\ Email: \{M.J.Coombes@lboro.ac.uk,W.Chen@lboro.ac.uk,P.M.Render@lboro.ac.uk\}
}

\begin{abstract}
This paper details a method to ascertain the reachability of known emergency landing sites for any fixed wing aircraft in a forced landing due to engine failure in steady uniform wind conditions. With knowledge of the aircraft's state and parameters, and landing site location and landing direction, the minimum height loss path can be defined. This uses glide performance calculations and a trajectory planner to give a minimum height loss to each landing site. Based on the aircraft's initial altitude it can calculate if the site is reachable, and how reachable it is. The path definition takes into account wind and uses a geometric shape called a trochoid to define the gliding turns in wind. This method is generic enough for use by any aircraft in any wind conditions.
\end{abstract}

Index Terms-Reachability Analysis; Gliding; Forced landings; Trochoids.

\section{Introduction}

The operation of Unmanned Aerial System (UAS) by the military has increased over the last couple of decades. This is because they offer advantages over the operation of manned aircraft. As they don't have to be designed to hold pilots, they have much longer endurance, and have larger payload capacities. The main advantage is that it removes the operator of the aircraft from danger. This makes them highly attractive for long surveillance or strike missions in dangerous areas, while the use of manned aircraft is expensive and requires skilled pilots.

The reasons that the military are increasingly using UASs are the same reasons driveing interest in their use for civilian applications. Some of the uses include search and rescue, border security, law enforcement, pipe line inspection, aerial photography, and environmental monitoring. Previously the military have large budgets and were the only organisations that could initially afford these expensive systems. Due to the recent miniaturisation of electronics and increasing maturity, UAS are becoming small and cheap enough for the civilian market.

By removing the pilot, a number of safety issues are introduced, chief among which is how a UAS would deal with an engine failure, and carry out a forced landing. As UASs tend to primarily be single engined fixed wing aircraft, they are as vulnerable to forced landings due to engine failure as any single engine General Aviation (GA) aircraft. Emergency landing after an engine failure was the second largest killer of pilots in 2001 in the US. Out of 298 fatal accidents involving GA aircraft, 46 were caused by engine failure, second only to loss of aircraft control [1]. This is why the forced landing situation is a major stumbling block to UAS integration in to National Airspace System (NAS), and why a contingency management system is important [2].

Upon an engine failure, the aircraft must carry out what is known as a forced landing. The aircraft, now unpowered, is effectively a glider and will need to land at an unprepared location. These landing sites are normally fields, many of which may be unsuitable due to size or obstacles. These are complex situations which require a great deal of practice for a human pilots to master. A lot of information is needed and a number of very complex decision must be made in order to perform a successful forced landing. Firstly an engine failure needs to be identified. Then a list of landing sites is required which can come from a number of sources, such as Geographic Information System (GIS), map data, presurveys, or live computer vision techniques. The list needs to be narrowed down to sites that are within the range of the aircraft, which requires the aircraft's glide performance and a minimum glide path to be known. Using data pertaining to this trimmed list of landing sites, a decision needs to be made on which of these site to aim to land in. A path then needs to be planned to the chosen landing site from the aircraft's current position that will get the aircraft lined up with the site at the correct altitude while accounting for wind an other uncertainties. Once the aircraft is on approach to the chosen site, a landing has to be performed.

The reachability of landing sites has been an area neglected in previous research. This is of paramount importance as there is very little point at aiming to land at the most perfect landing site if you are unable to reach it. Therefore it is necessary to be able to calculate each known landing site's reachability. It is also important to show how reachable a landing site is, which is why the notion of excess glide range is introduced. To calculate this, the aircraft's glide performance, the minimum height loss path to fly, and path of the approach are all needed. References [3] and [4] lay out a number of equations for working out an aircrafts glide performance, as well as explaining the maneuvers and speeds to minimse a gliding aircrafts height loss. The work is extended [5] and [6].

A method is presented in [3] to calculate the reachability of a landing site. It uses a human forced landing approach technique called the 'high-key low-key' technique [7]. This, 
combined with maximum glide range equations can show the excess glide range of the aircraft for a selected landing site. The high-key low-key technique is intended to give a human pilot good visibility to study the site and time to prepare the aircraft for landing. This results in inefficient use of the available glide range. As well not being applicable to larger faster aircraft a more generic technique was required.

Dubins showed that a minimum path length exists only when there are maximum curvature circular arcs connected by a straight segments, with a maximum of 3 segments [8]. Which in this context means a path taken by the aircraft must contain two turns at maximum turn rate, connected by a straight glide path between them which makes a perfectly smooth trajectory. So upon engine failure the aircraft will turn on to a track which after a level glide will intercept the turn to final (final landing decent path to runway) at 500ft. For a normal landing the turn to final should not be below 500ft [9]. This can be used for any aircraft or runway orientation, also for any radius of turn. This will minimise height loss for the aircraft, and work for an aircraft of absolutely any performance.

However these circular flight paths do not take into consideration the effects of wind, which is very important for a gliding aircraft. The concept of Dubins curves is extended to take in to account for wind, so instead of the straight glide being tangential to two circles, they will be tangent to two geometric shapes called trochoids. How trochoids are used for path planning in the presence of wind are demonstrated in [10] and [11]. This paper will extend these further to show how this can be used as a glide path definition for a landing sites reachability calculation.

In Section 2 the equations describing the glide performance of an aircraft in straight and level and turning flight are defined. Presented in Section 3 are a set of equations to define a minimum height loss path in the presence of wind. Section 4 shows how the trochoidal path can be used with the glide performance equations to ascertain if a particular landing site is reachable and how much excess glide distance it has when it reaches it. The proposed method is demonstrated in Section 5 by a forced landing scenario of a fast low drag aircraft. Finally Section 6 makes some concluding remarks.

\section{Glide performance}

Before any glide paths or approach paths are defined, the glide performance of the aircraft needs to be known. The vertical sink $\left(V_{s}\right)$ for a given airspeed is needed. The aircrafts Lift/Drag ratio is the glide ratio of aircraft, and a number of factors will influence both of these, the main being the aircrafts drag polar.

Calculations are put forward in [3] to calculate the glide ratio and vertical sink of a gliding aircraft based on its parameters. The calculation for $V_{s}$ presented assumes that the aircraft remains at best glide speed constantly throughout the flight. The best glide speed is the airspeed that the aircraft needs to fly at which maximises its glide ratio. How this is calculated is shown in [3]. However in a co-ordinated turn, this speed increases due to the higher levels of lift and therefore increases drag from the greater normal loading (n). It is assumed that the aircraft would speed up in turns to maintain best glide for these new conditions. This is not what an aircraft would actually do and the airspeed transition would add inaccuracies in to the equations, so a modified equation is shown here that gives the vertical sink of the aircraft for any airspeed, not simply the optimum.

For an aircraft with no forward thrust, to maintain airspeed the aircraft must remain in equilibrium by balancing out the drag force with a component from its weight. As such, the vertical speed can be calculated by

$$
V_{s}=\frac{D V}{W}
$$

where $D$ is drag, $V$ is airspeed and $W$ is the weight of the aircraft.

Using Eq. (1) with an aircraft's parabolic drag polar, $V_{s}$ can be calculated in straight and level flight as shown in [3] the vertical speed can be calculated by

$$
V_{s}=A V^{3}+\frac{B}{V}
$$

with

$$
A=\frac{0.5 \rho_{0} S C_{D o}}{W}
$$

and

$$
B=\frac{2 W}{\rho_{0} S \pi A_{r} e}
$$

$A_{r}$ is the aspect ratio, $\rho_{0}$ is the density of air at sea level, $C_{D o}$ is the drag at zero lift, $e$ is oswald efficiency factor, and $S$ is the wing area. It is assumed aircraft instantaneously attains the desired bank angle.

For a co-ordinated turning maneuver, the sink increases as $n$ increases from unity, meaning that $L=n W$. This can be substituted in to Eq. (1) to give Eq. (5).

$$
V_{s \phi}=\frac{D V n}{L}
$$

where $V_{s \phi}$ is the vertical sink in a turn.

[3] shows that normal loading in a turn is equivalent to $\sec (\phi)$ where $\phi$ is the bank angle. Substituting $C_{L}$ for lift and the parabolic drag polar $C_{D}=C_{D o}+\frac{C_{L}^{2}}{\pi e A_{r}}$ in to Eq. (5), the full equation for sink in a turn for any airspeed or bank angle is given by

$$
V_{s \phi}=A V^{3}+\frac{B \sec ^{2}(\phi)}{V}
$$

\section{Trochoidal turn path}

A turn at a constant velocity and bank angle in no wind (assuming instantaneous roll) will be a perfect circle. An initial turn circle and a final turn circle linked by a tangent would define the minimum height loss path in no wind. In the prescence of wind, the aircraft is no longer in the Earth frame, but in the wind frame. This means the circle becomes a shape known as a trochoid. Tangents will be calculated to link the first and second trochoidal turn path into a single smooth trajectory.

A trochoid is a curve traced by a point on a radius of a circle where the centre of the circle is moving at a fixed rate 


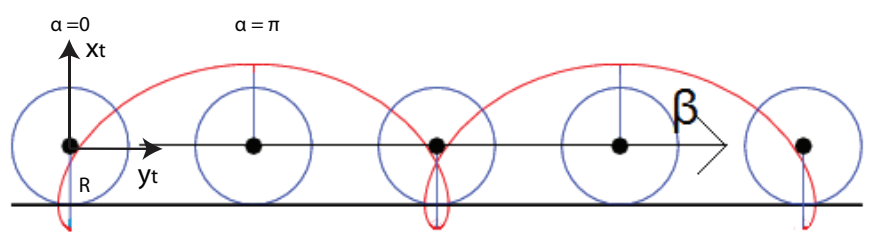

Figure 1: A circle moving at a fixed rate $\beta$, while tracing a trochoid at a point at a fixed radius

$(\beta)$ along a straight line. This is shown in Fig. 1. The shape can be defined parametrically by angle $(\alpha)$ as the input in Eq. (7). $\alpha$ can be related to the heading of the aircraft, but as it is used slightly differently between the initial and final trochoids, for the moment it can be thought of as heading change. How it can be used to ascertain the heading will be explained later.

$$
\begin{array}{r}
\quad x_{t}=-R \cos (\alpha) \\
y_{t}=R \sin (\alpha)+R \beta \alpha
\end{array}
$$

The aircraft flies a circular path in air but as this is in the wind axis, the transformation to Earth axis means that the aircraft is in fact flying a trochoidal path. This is because the turn circle centre is being blown down wind at the rate $\beta$ which is the ratio of windspeed to the aircraft's airspeed, shown in Eq. (8).

$$
\beta=\frac{V_{w}}{V}
$$

where $V_{w}$ is the wind velocity.

Eq. (7) is the parametric equation for a turn circle with radius $R$ but the $R \beta \alpha$ term represents the aircraft getting blown down wind during its turn and it is the term causes the path to be trochodial.

The trochoidal path method described in [10] has been adapted for use here. However the the aim of this paper is to seek a time optimal path for a given set of initial and final conditions, all their calculations are based on time. Angles are more useful in the forced landing application, as it can be directly related to the track angle of the aircraft. All equations are re-derived to use angle, and further deviations are different from [10].

\subsection{Trochoid definitions and positions}

The trochoid is defined in the trochoidal frame, which is where the $y$ direction is aligned with the wind. This means the equations are much simpler. However the trajectories in the trochoidal frame will have to be rotated to put them in the Earth frame after their calculation. Shown in Fig. 2 is a trochoid in the trochoidal frame with the aircraft turning in the clockwise direction through a heading of $0 \rightarrow 4 \pi$. A positive $\dot{\psi}$ is a turn in the clockwise direction.

The path for the whole flight must be defined with only the initial, and final conditions of the aircraft. These include initial aircraft heading $\left(\psi_{0}\right)$, final aircraft heading which is the runway landing direction $(\Gamma)$, the position of the turn to final waypoint $\left(F_{x}, F_{y}\right)$ which is also defined relative to the aircraft's starting location $x_{r}$ and $y_{r}$. The

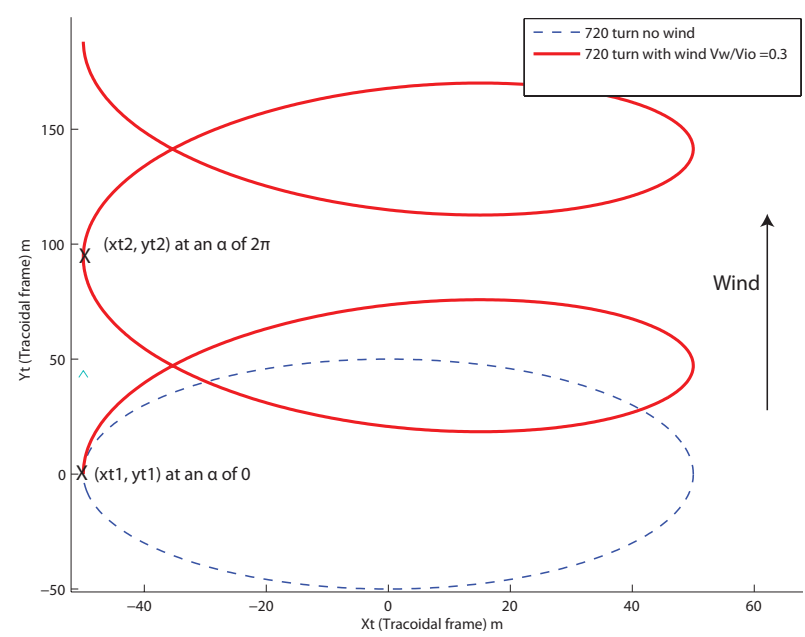

Figure 2: $4 \pi$ turn with and without wind in the trochiodal frame. The wind is 0.3 of the aircrafts airspeed $(\beta=0.3$ where $\mathrm{R}=50 \mathrm{~m}$ which can be obtained from $\mathrm{V} / \dot{\psi}$ )

turn to the final waypoint is calculated in Eq. (10), where the waypoint is defined relative to the centre line of the runway at a longitudinal distance that would enable the aircraft to glide to the runways centre from a height of 500ft.It is then transformed into the global co-ordinates by rotating the runway direction.

$$
\left[\begin{array}{l}
F_{x} \\
F_{y}
\end{array}\right]=\left[\begin{array}{cc}
\cos \Gamma & -\sin \Gamma \\
\sin \Gamma & \cos \Gamma
\end{array}\right]\left[\begin{array}{c}
0 \\
0.3048 \times 500 \gamma
\end{array}\right]
$$

where $0.3048 \times 500 \gamma$ is the distance an aircraft will travel from a height of $500 \mathrm{ft}$ at a particular glide ratio $(\gamma)$.

As $\left(F_{x}, F_{y}\right)$ are in the Earth frame and need to be rotated into the trochodial frame, they must be rotated by the wind direction $\left(\psi_{w}\right)$ shown by

$$
\left[\begin{array}{l}
F_{t x} \\
F_{t y}
\end{array}\right]=\left[\begin{array}{cc}
\cos \psi_{w} & -\sin \psi_{w} \\
\sin \psi_{w} & \cos \psi_{w}
\end{array}\right]\left[\begin{array}{l}
F_{x} \\
F_{y}
\end{array}\right]
$$

There will be two trochoids; one for the initial turn and one for the final turn. The equations for these need to be defined using the initial and final conditions. The radius of the turn is defined as

$$
R=\frac{V}{\dot{\psi}}
$$

where $\delta$ is the sign of the turn direction.

The angle $\alpha$ needs to be adjusted by the phase angle $\eta$ to account for the transformation between frames, and for the initial and final headings. These phase angles are defined in Eq. (12).

$$
\eta_{1}=\psi_{0}-\psi_{w}, \quad \eta_{2}=\Gamma-\psi_{w}-\delta_{2} 2 \pi
$$

where $\eta_{1}$ for the initial turn and $\eta_{2}$ for the final turn. The term $\delta_{2} 2 \pi$ is added to the second turn as the aircraft needs to be at $\Gamma$ at the end of the trochoid, and the end of the trochoid is at $2 \pi$.

The start of the first trochoid must be placed at the point where the aircraft starts $\left(x_{0}, y_{0}\right)$. It is easier however to have the aircraft start at $(0,0)$ which will be used here. 


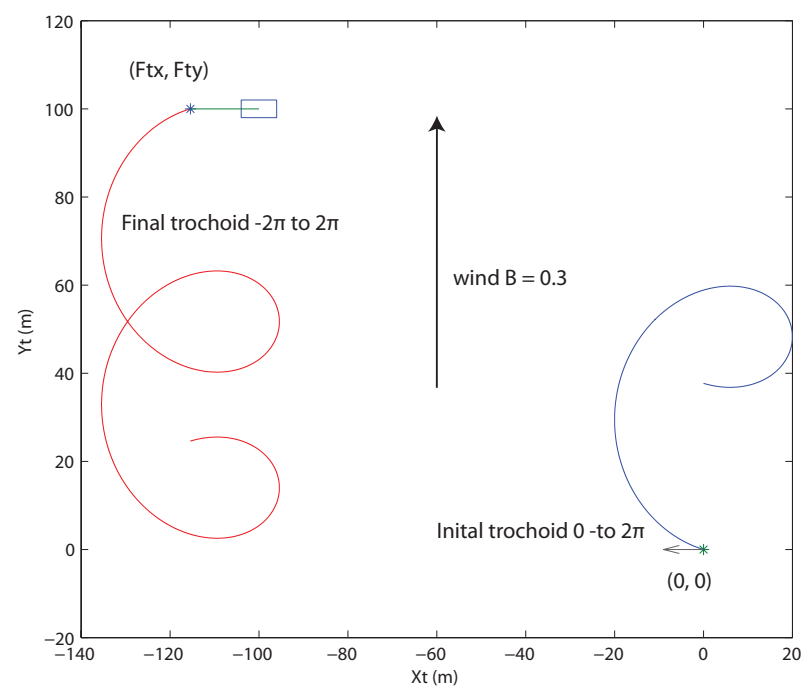

(a) Trochoidal frame

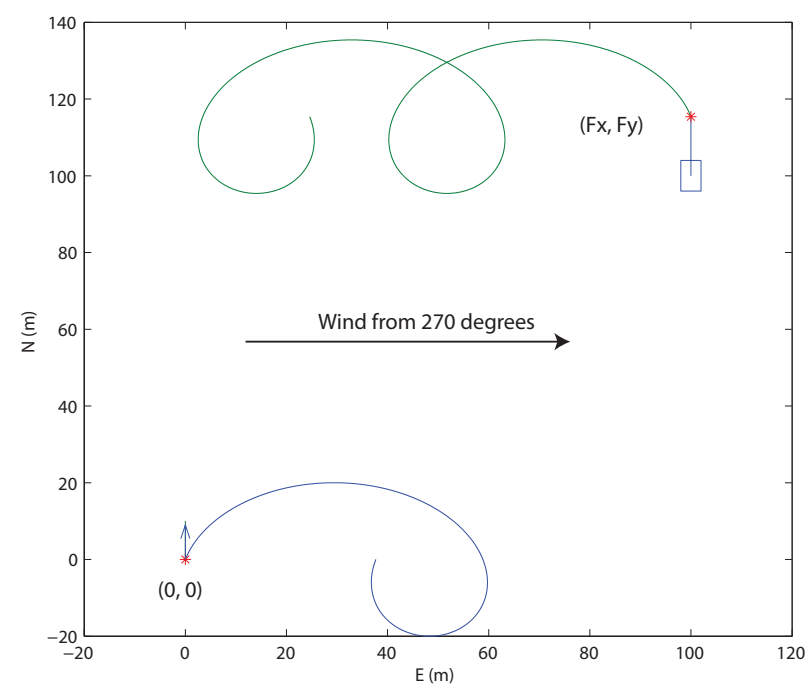

(b) Earth frame

Figure 3: Position of both trochoids in the trochoidal and earth frame. As the initial conditions have been rotated to the trochoidal frame, so once the paths are defined the can simply be rotated about the starting point of the aircraft

Similarly the end of the second trochoid must be located at the position of the turn to final waypoint. They are repositioned by calculating the co-ordinates of the start of the initial trochoid. and the end of the final trochoid.

Following Eq. (7) and (12), the initial and final trochoids are shown in Eq. (13) and Eq. (14), respectively.

$$
\begin{gathered}
x_{t 1}=\frac{V}{\delta_{1} \dot{\psi}} \cos \left(\eta_{1}\right)+x_{0} \\
y_{t 1}=-\frac{V}{\delta_{1} \dot{\psi}} \sin \left(\eta_{1}\right)+y_{0} \\
x_{t 2}=\frac{V}{\delta_{1} \dot{\psi}} \cos \left(\eta_{2}\right)+F_{t x} \\
y_{t 2}=\frac{V}{\delta_{1} \dot{\psi}} \sin \left(\eta_{2}\right)+2 \frac{V}{\delta_{2} \dot{\psi}} \beta \pi+F_{t y}
\end{gathered}
$$

where $\left(x_{t 1}, y_{t 1}\right)$ moves the start of the initial trochoid turn to $\left(x_{0}, y_{0}\right)$ and $\left(x_{t 2}, y_{t 2}\right)$ moves the end of the second trochoid to the landing site approach point $\left(F_{t x}, F_{t y}\right)$.

Eq. (11), (12), and Eq. (13) or Eq. (14) can be substituted into Eq. (7) to give the equation for the initial and final turn trochoid in the trochoidal frame. As shown in Eq. (15), and (16) below

$$
\begin{array}{r}
x_{t}=-\frac{V}{\delta_{1} \dot{\psi}} \cos \left(\alpha+\eta_{1}\right)+x_{t 1} \\
y_{t}=\frac{V}{\delta_{1} \dot{\psi}} \sin \left(\alpha+\eta_{1}\right)+\frac{V}{\delta_{1} \dot{\psi}} \beta \alpha+y_{t 1} \\
x_{t}=-\frac{V_{i o}}{\delta_{2} \dot{\psi}} \cos \left(\alpha+\eta_{2}\right)+x_{t 2} \\
y_{t}=\frac{V_{i o}}{\delta_{2} \dot{\psi}} \sin \left(\alpha+\eta_{2}\right)+\frac{V_{i o}}{\delta_{2} \dot{\psi}} \beta \alpha+y_{t 2}
\end{array}
$$

Each of the two turns can be in either direction. These directions are represented by $\delta_{1}, \delta_{2}$ with are either -1 for an anti clockwise direction, and 1 for clockwise, where there are four possible trajectories.

How the two trochoids are positioned, plotted, and then rotated is shown in Fig. 3. The initial and final trochoid are plotted relative to the the aircrafts initial position and the final waypoint $\left(F_{t x}, F_{t y}\right)$ in the trochoidal frame. Everything is then rotated to be in the Earth frame. The initial and final conditions in Fig. 3 are shown below

$$
\begin{array}{cc}
\psi_{0}=0 & \Gamma=\pi \\
y_{0}=0 & \psi_{w}=\frac{\pi}{2}
\end{array} \quad F_{x}=100 \quad F_{y}=115.4 \quad x_{0}=0
$$

where he wind must be converted into the direction of travel, so in this case the wind is from $\frac{3 \pi}{2}$ so travels at $\frac{\pi}{2}$

For an optimal path the total angle change over both turns must be less than $4 \pi$ which is proven in [10]. This is why the initial trochoid one is plotted between $0 \rightarrow 2 \pi$, and the final trochoid is plotted between $-2 \pi \rightarrow 2 \pi$. For the trajectory to be optimal the tangent will lay somewhere between these two trochoids.

\subsection{Finding tangents between trochoids}

The two trochoids now need to be connected with a straight and level glide between them which will create a smooth trajectory. The departure point from the initial trochoid will be referred to as $P_{a}$, which will occur at angle $\alpha_{a}$. Similarly at the end of the straight glide, the join point on the final trochoid is referred to as $P_{b}$ at an angle of $\alpha_{b}$. It will be these two angles which must be found to define the whole path. This can be seen in Fig. 4.

For a tangent to exist the aircraft's ground track $\left(\psi_{g}\right)$ at both point $P_{a}$ and $P_{b}$ must be the same, as well as their velocity vectors shown in Eq. (18) and (19). Both $\alpha_{a}$ and $\alpha_{b}$ need to be found so the $(x, y)$ co-ordinates for $P_{a}$ and $P_{b}$ can be found from Eq. (13), and (14).

$$
\begin{aligned}
\tan \left(\psi_{g}\right) & =\frac{y\left(\alpha_{b}\right)-y\left(\alpha_{a}\right)}{x\left(\alpha_{b}\right)-x\left(\alpha_{a}\right)} \\
\tan \left(\psi_{g}\right) & =\frac{\dot{y}\left(\alpha_{b}\right)}{\dot{x}\left(\alpha_{b}\right)}=\frac{\dot{y}\left(\alpha_{a}\right)}{\dot{x}\left(\alpha_{a}\right)}
\end{aligned}
$$




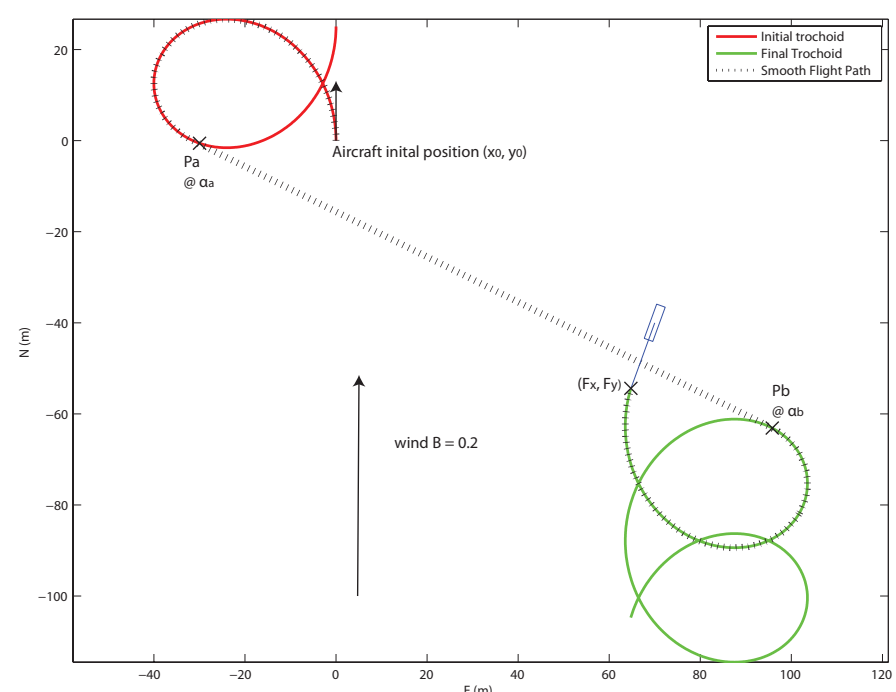

Figure 4: Anti-clockwise clockwise flight path, where both trochoids and the optimal tangent between them have been defined. The tangent starts at $P_{a}$ and finishes as $P_{b} . \psi_{0}=0$ $\Gamma=\frac{\pi}{6}$

By substituting Eq. (15) and (16), into Eq. (18) a full equations for the heading of the tangent can be found in Eq. (20) (see next page).

The relationship between $\alpha_{a}$ and $\alpha_{b}$ needs to be found in order for $\alpha_{a}$ to be substituted for $\alpha_{b}$ in Eq. (20) to put it in terms of only $\alpha_{a}$ so the equation can be solved to only a single variable. The total angle travelled by the aircraft around both turns must be equal to $\psi_{0}-\Gamma$ as this is the minimum angle to get the aircraft from the initial heading to the final heading. The angle $\alpha_{a}$ is the total angle subtended around the curve over the first trochoid by the aircraf,t i.e. the heading change of the aircraft around the first turn. As the aircraft travels the opposite direction around the second trochoid, and is positioned relative to the end of the trochoid $(2 \pi), \alpha_{b}$ is not the angle subtended by the aircraft around the second trochoid. The angle actually subtended is $\alpha_{b}^{\prime}$ so $\alpha_{a}+\alpha_{b}^{\prime}$ is equal to the total angle travelled by the aircraft around both turns. As a trochoid is based on a cyclical function there will be an infinite number of tangents between both trochoids. Only solutions within a certain range are wanted to keep the path optimal, but there may be multiple solutions within this range so to find them, multiples of $2 k \pi$ will be added where $k \in[-3,-2,-1,0,1,2]$. The effect of $\mathrm{k}$ is shown in Fig. 5. Increasing $\mathrm{k}$ by 1 shifts the angle of tangency to the next loop on the final trochoid. Eq. (21) can be formulated to give a starting point for relating $\alpha_{a}$ and $\alpha_{b}$.

$$
\alpha_{a}+\alpha_{b}^{\prime}=\psi_{0}-\Gamma+2 k \pi
$$

The actual angle subtended is $\alpha_{b}^{\prime}=\delta_{2} 2 \pi-\alpha_{b}+2 k \pi$. An example of this is shown in Fig. 6. By substituting this in to Eq. (21) an equation that fully defines the relationship between $\alpha_{a}$ and $\alpha_{b}$ is shown in Eq. (22)

$$
-\delta_{1} \alpha_{a}+\delta_{2} \alpha_{b}-\delta_{2} 2 \pi+2 k \pi=\psi_{0}-\Gamma
$$

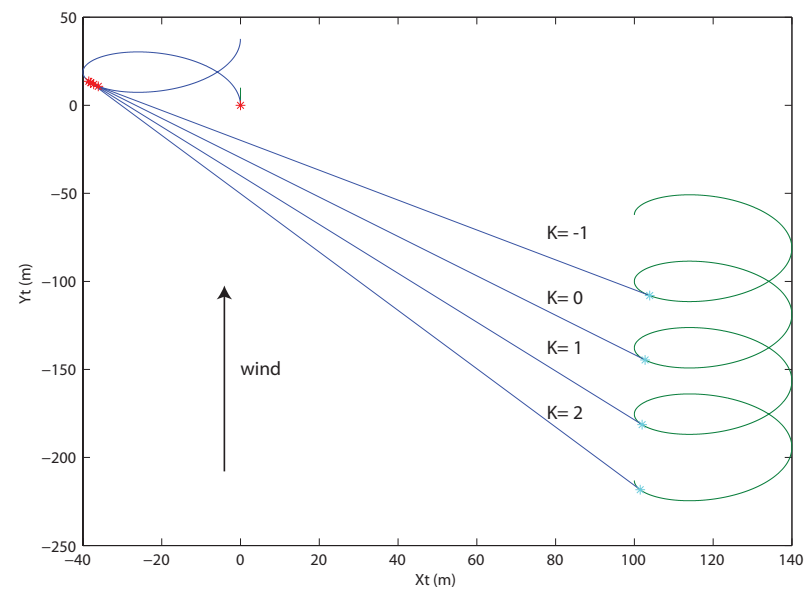

Figure 5: The effect of $\mathrm{k}$

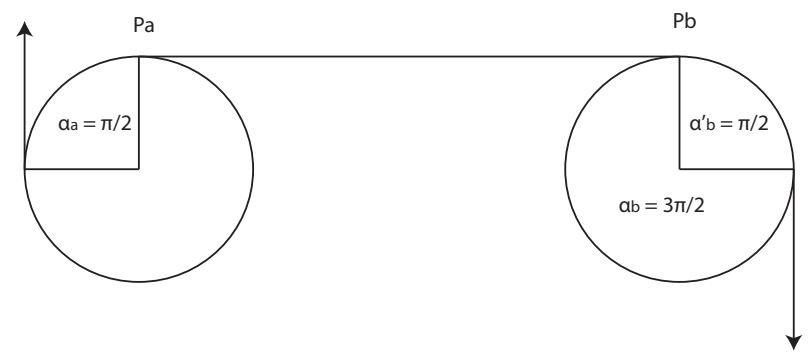

Figure 6: Shows the relationship between $\alpha_{a}, \alpha_{b}$, and $\alpha_{b}^{\prime}$

This can be simplified and the phase angle's $\eta_{1}$ and $\eta_{2}$ can be substituted in. This is shown below

$$
\alpha_{b}=\frac{\delta_{1}}{\delta_{2}} \alpha_{a}+\frac{\eta_{1}-\eta_{2}+2 k \pi}{\delta_{2}}
$$

A full equation for the angle of tangency between the two trochoids is needed. The $\alpha_{b}$ terms from Eq. (20) can be substituted for $\alpha_{a}$ using Eq. (23), the result is shown in Eq. (24) (see next page).

Now by equating Eq. (24), with Eq. (20) which removes the $\tan \left(\psi_{g}\right)$ term, and by using the identity $\sin (\psi)^{2}+$ $\cos (\psi)^{2} \equiv 1$ the whole equation can be simplified to Eq. (25).

$$
\begin{gathered}
V\left(V_{w} \frac{\delta_{1}-\delta_{2}}{\delta_{1} \delta_{2} \dot{\psi}}-\left(x_{t 2}-x_{t 1}\right)\right) \cos \left(\delta_{1} \psi_{1}+\eta_{1}\right) \\
+V\left(\left(y_{t 2}-y_{t 1}\right)+\frac{V_{w}}{\dot{\psi}}\left(\psi_{1}\left(\frac{\delta_{1}}{\delta_{2}}-1\right)+\frac{\eta_{1}-\eta_{2}+2 k \pi}{\delta_{2}}\right)\right) \\
\sin \left(\left(\delta_{1} \psi_{1}+\eta_{1}\right)\right)-V_{w}\left(x_{t 2}-x_{t 1}\right)-\frac{V^{2}\left(\delta_{2}-\delta_{1}\right)}{\delta_{2} \delta_{1} \dot{\psi}}=0
\end{gathered}
$$

There is no analytical solution for $\alpha_{a}$ as this equation is transcendental.

\subsection{Solving for same side turns}

As both turns can be in either directions, there are four different combinations. When the turns are on the same side, i.e. clockwise/clockwise or anti-clockwise/anti-clockwise, this means $\delta_{1}=\delta_{2}$. Consequently Eq. (24) can be simplified to Eq. (26). The extra term $2 \pi m$ has been added. It works similarly to $\mathrm{k}$ but instead of shifting the point $P_{b}$ to the next 


$$
\tan \left(\psi_{g}\right)=\frac{\frac{V}{\delta_{2} \dot{\psi}} \sin \left(\alpha_{b}+\eta_{2}\right)+\frac{V}{\delta_{2} \dot{\psi}} \beta \alpha_{b}+y_{t 2}-\frac{V}{\delta_{1} \dot{\psi}} \sin \left(\alpha_{a}+\eta_{1}\right)+\frac{V}{\delta_{1} \dot{\psi}} \beta \alpha_{a}+y_{t 1}}{-\frac{V}{\delta_{2} \dot{\psi}} \cos \left(\alpha_{b}+\eta_{2}\right)+x_{t 2}+\frac{V}{\delta_{1} \dot{\psi}} \cos \left(\alpha_{a}+\eta_{1}\right)+x_{t 1}}
$$

$$
\tan \left(\psi_{g}\right)=\frac{\left(x_{t 2}-x_{t 1}\right)+V \frac{\delta_{2}-\delta_{1}}{\delta_{2} \delta_{1} \dot{\psi}} \cos \left(\delta_{1} \psi_{1}+\eta_{1}\right)}{\frac{\delta_{2}-\delta_{1}}{\delta_{2} \delta_{1} \dot{\psi}} \sin \left(\delta_{1} \psi_{1}+\eta_{1}\right)+\left(y_{t 2}-y_{t 1}\right)+\frac{V_{w}}{\dot{\psi}}\left(\psi_{1}\left(\frac{\delta_{1}}{\delta_{2}}-1\right)+\frac{\eta_{1}-\eta_{2}+2 k \pi}{\delta_{2}}\right)}
$$

trochoidal loop on the second trochoid it shifts both $P_{a}$ and $P_{b}$ to the next loop on both trochoids. As there are infinite solutions, so both $\mathrm{k}$ and $\mathrm{m}$ must be changed to in order to find to optimum path. As $\psi_{g}=\alpha_{a}, \alpha_{a}$ can be found.

$$
\tan \left(\alpha_{a}\right)=\frac{\left(x_{t 1}-x_{t 1}\right)}{\left(y_{t 2}-y_{t 1}\right)+V\left(\frac{\eta_{1}-\eta_{2}+2 k \pi}{\delta_{2}}\right)}+2 \pi m
$$

Eq. (26) can be easily solved for $\alpha_{a}$, and by using Eq. (23), $\alpha_{b}$ can be found. A range of values for $\mathrm{k}$ and $\mathrm{m}$ need to be used to find the path that satisfies the conditions in Eq. (27) which will make the path optimal.

$$
\left\{\begin{array}{c}
0<\alpha_{a}<2 \pi \\
-2 \pi<\alpha_{b}<2 \pi
\end{array}\right.
$$

\subsection{Solving numerically for different side turns}

As $\delta_{1} \neq \delta_{2}$ Eq. (25) must be used to find the tangent angle $\alpha_{a}$ from the first trochoid to the second. The roots $\alpha_{a}$ in the equation need to be found between the range $0 \rightarrow 2 \pi$, as $\alpha_{a}$ needs to be in this range to make the trajectory optimum. The roots can be found when $f\left(\alpha_{a}\right)=0$, and any root finding technique can be used. Here the bisection method is used. A good introduction to the bisection method is contained in [12]. The bisection method finds all the values of $x$ where $f(x)=0$ between an interval $[a, b]$. If $f(a)$ and $f(b)$ have opposite signs, this means that they bracket a root, and it is on this principle that the algorithm works.

As mentioned previously, a range of $k[-1,0,1,2,3]$ values need to be used in order to find the best tangent to make the flight path as short as possible.

In the interval $[a, b]$ (for a given value of $k$ ) there maybe multiple roots, but some of these will create sub optimal paths, or unfeasible flight paths. Now that a range of exit angles are known for the first trochoid $\left(\alpha_{a}\right)$, these will be narrowed down to the shortest legitimate flight path. To eliminate the invalid paths, the angle of the tangent from $P_{a}$ to $P_{b}$ will be compared to the track of the aircraft at point $P_{a}$. To find the aircrafts track the aircrafts $x$ and $y$ velocities can be used with the 4-quadrant inverse tangent function $(\operatorname{atan} 2)$. The aircraft velocities are obtained from differentiating Eq. (15) to give Eq. (28).

$$
\begin{array}{r}
V_{t x a}=V_{i o} \sin \left(\alpha_{a}+\eta_{1}\right) \\
V_{t y a}=V_{i o} \cos \left(\alpha_{a}+\eta_{1}\right)+V_{w}
\end{array}
$$

where $V_{t x a}$ and $V_{t y a}$ are the aircraft $x$, and $y$ velocities in the trochodial frame at point $P_{a}$. All these checks will be performed in the trochoidal frame for simplicity.

The track of the aircraft can be calculated by

$$
\begin{gathered}
\psi_{t g}=\operatorname{atan}\left(\frac{V_{x t a}}{V_{y t a}}\right)=\operatorname{atan}\left(\frac{V \sin \left(\alpha_{a}+\eta_{1}\right)}{V \cos \left(\alpha_{a}+\eta_{1}\right)+V_{w}}\right) \\
\theta_{t a n g}=\operatorname{atan}\left(\frac{x_{t P b}-x_{t P a}}{y_{t P b}-y_{t P a}}\right)
\end{gathered}
$$

where $\left(x_{t P a}, y_{t P a}\right)$ is the co-ordinate of $P_{a}$ at the point tangent meets the first trochcoid, and $\left(x_{t P a}, y_{t P b}\right)$ the coordinates at point $P_{b}$. $\theta_{\text {tang }}$ is the angle of the tangent in the trochoidal frame from $P_{a}$ to $P_{b}$.

If $\psi_{g}=\theta_{\text {tang }}$, then that particular flight path is valid. As can be seen in Fig. 7, the invalid path has a $\psi_{g}=91^{\circ}$ but the tangent is $180^{\circ}$ off at $\theta_{\text {tang }}=271^{\circ}$. The shortest path out of the valid ones has the largest $\alpha_{b}$ angle. This is because the closer to $2 \pi \alpha_{b}$ is, the smaller the angle the aircraft will have to subtend around the second trochoid. This can be clearly seen in Fig. 7 the two valid paths show their $\alpha_{b}$ values and the path labelled as the shortest has a much higher $\alpha_{b}$ value than the other. As the angle $\alpha_{a}$ is constrained to between $0 \rightarrow 2 \pi$ this has been already be optimised for a given $\alpha_{b}$.

\section{Excess glide, and arc length calculations}

After the trajectory has been defined, the distance travelled, the height lost, and excess glide now need to be calculated. To find the distance travelled, the arc length (L) needs to be calculated for both turns, as well as the straight glide. As the turn is now defined by a trochoid, a new equation is needed for arc length. For parametric equations arc length is defined below by

$$
L=\int \sqrt{\frac{d x^{2}}{d \psi}+\frac{d y^{2}}{d \psi}} d \psi
$$

Differentiating Eq. (7) with respect to $\psi$ gives

$$
\begin{array}{r}
\frac{d x}{d \psi}=\delta R \cos (\psi) \\
\frac{d y}{d \psi}=\delta R \sin (\psi)+\delta R \beta
\end{array}
$$

Substituting Eq. (32) in to Eq. (31) yields Eq. (33). 


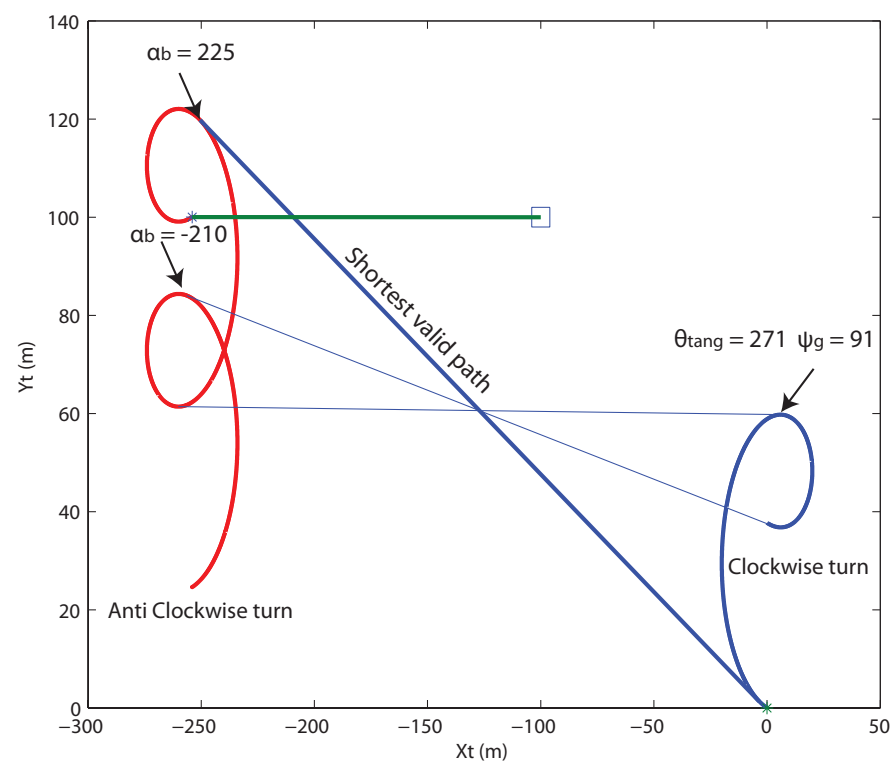

Figure 7: There are 3 tangents between both trochoids for $\mathrm{k}=0$, one path is not a legitimate flight path, one is suboptimal, the other is tangent chosen as the flight path as it give the shortest distance.

$\sqrt{\frac{d x^{2}}{d \psi}+\frac{d y^{2}}{d \psi}}=R \sqrt{\sin (\psi)^{2}+\cos (\psi)^{2}+2 \beta \cos (\psi)+\beta^{2}}$

which can be simplified to give the full integral for arc length shown below

$$
L=\int_{0}^{\psi} R \sqrt{1+2 \beta \cos (\psi)+\beta^{2}}
$$

Eq. (34) cannot be solved analytically but as these are a common type of integration a set of functions exist to deal with them. The functions are called elliptic integrals and are laid out in [13]. The particular function is an incomplete elliptic integral of the second kind which is defined in Eq. (35).

$$
E(\psi \mid m)=\int_{0}^{\psi} \sqrt{1-m \sin (\psi)^{2}} d \psi
$$

Although this is not in the exact same form as Eq. (34), by rearranging the input into this function, one can show

$$
\int_{0}^{\psi} R \sqrt{1+2 \beta \cos (\psi)+\beta^{2}}=2 R(1+\beta) E\left(\frac{\psi}{2} \mid \frac{4 \beta}{(1+\beta)^{2}}\right)
$$

Shown in Eq. (37) and (38) are the full definite integrals with the correct limits for the arc lengths for both trochoids.

$$
L_{a}=2 R(1+\beta) E\left(\frac{\alpha_{a}}{2} \mid \frac{4 \beta}{(1+\beta)^{2}}\right)-2 R(1+\beta) E\left(0 \mid \frac{4 \beta}{(1+\beta)^{2}}\right)
$$

where $L_{a}$ is the arc length subtended by the aircraft around the first trochoid.

$L_{b}=R(1+\beta) E\left(2 \pi \mid \frac{4 \beta}{(1+\beta)^{2}}\right)-2 R(1+\beta) E\left(\frac{\alpha_{b}}{2} \mid \frac{4 \beta}{(1+\beta)^{2}}\right)$

where $L_{b}$ is the arc length subtended by the aircraft around the second trochoid.

The total ground distance covered by the aircraft is shown in Eq. (39).

$$
L=L_{a}+L_{b}+\sqrt{\left(y_{t P b}-y_{t P a}\right)^{2}+\left(x_{t P b}-x_{t P a}\right)^{2}}
$$

Height loss around the turn needs to be calculated so the landing site's reachability and excess glide range can be ascertained. Knowing the glide ratio and glide distances, height loss across a glide can be calculated in still wind. When factoring in wind, the aircraft's ground speed is not constant around the turn, which means glide ratio is also not constant. As the vertical sink of the aircraft is calculated in the wind frame the height loss in the turn can be found from time in the turn.

As it is assumed that the aircraft can instantaneously achieve a given roll angle, meaning that the time taken by the aircraft subtending an angle can be calculated by using the aircrafts yaw rate $\dot{\psi}$. This calculation is shown in Eq. (40) where $\frac{V}{R}=\dot{\psi}$. While ground speed is not constant, the aircraft sink will be constant as a steady airspeed is being maintained.

$$
t_{a}=\frac{V}{R} \alpha_{a}
$$

where $t_{a}$ is the time the aircraft takes to travel around the first turn.

The time taken around the second turn $\left(t_{b}\right)$ is dealt with in Eq. (41) which is a modified version of Eq. (23).

$$
t_{b}=\frac{2 \pi}{\dot{\psi}}-t_{a}-\frac{\eta_{1}-\eta_{2}+2 k \pi}{\delta_{2} \dot{\psi}}
$$

$t_{a}$ and $t_{b}$ can be used alongside Eq. (6) for aircraft sink to calculate height lost in the turn shown below

$$
\Delta h_{a}=V_{s \phi} t_{a}
$$

where $V_{s \phi}$ is the aircrafts vertical sink in a turn, $\Delta h_{a}$ the height lost around the first turn, and $t_{a}$ can simply be replaced with $t_{b}$ to get the height lost around the second turn.

The height loss in the straight and level glide between both turns $\left(\Delta h_{g}\right)$ is calculated below

$$
\Delta h_{g}=V_{s} \frac{\sqrt{\left(x_{t P b}-x_{t P a}\right)^{2}+\left(y_{t P b}-y_{t P a}\right)^{2}}}{\sqrt{V_{t y a}^{2}+V_{t x a}^{2}}}
$$

where the numerator represents the distance travelled over ground, the denominator is the aircraft's ground speed and $V_{s}$ is the sink calculated from Eq. (2).

The total loss of height over the entire trajectory $\left(\Delta h_{t o t}\right)$ is calculated by 


$$
\Delta h_{t o t}=V_{s \phi}\left(t_{a}+t_{b}\right)+\Delta h_{g}
$$

The excess glide range can now be calculated, as excess height can be found from $h-\Delta h_{t o t}$.

$$
E_{g}=\gamma\left(h-\Delta h_{t o t}-152\right)
$$

where $152 \mathrm{~m}$ is the height required to fly final to land, which is $500 \mathrm{ft}$.

\section{Results and discussion}

An example forced landing situation will now be considered. To show that this method for trajectory generation will work on any aircraft, an Airbus A320-400 will be used. This is a fast low drag aircraft that has very large turn radius, which would have made the previous method fail [3]. While the A320 is not a UAS and as it is also not single engined, the incident that made an American airlines A320 perform a forced landing into the Hudson River proves that it can happen to any aircraft. A flock of geese were the reason for its double engine failure, the report of this incident is contained in [14]. The author is in no way implying that the heroic actions of the pilots where wrong, merely using this as an example use of the algorithms on a extreme aircraft type (The river was the best choice regardless, due to the proximity to buildings if the A320 was to try and land back at LaGuardia). All the parameters needed for the glide calculations are contained in Table 1, and many of these parameters have been obtained from [15]. Trajectories, and glide predictions will be generated both with and without wind.

TABLE 1: Parameters for the A320-400

\begin{tabular}{ll}
\hline Parameter & Value \\
\hline Cdo (clean) & 0.022 \\
$\mathrm{~S}$ & $122.5 \mathrm{~m}^{2}$ \\
$A_{r}$ & 9.5 \\
$\mathrm{k}$ & 1.2992 \\
$\mathrm{~W}$ & $671108 \mathrm{~N}$ \\
$\mathrm{~A}$ & $2.460 \times 10^{-} 6$ \\
$\mathrm{~B}$ & 389.3 \\
$V_{\text {io }}$ & $112 \mathrm{~m} / \mathrm{s}$ \\
$\gamma$ & 16.2 \\
$V_{\text {sio }} \phi=45^{\circ}$ & $9.81 \mathrm{~m} / \mathrm{s}$ \\
\hline
\end{tabular}

The A320 has just taken off from a runway heading at $020^{\circ} 12.2 \mathrm{~km}$ north of the runway climbing past $1300 \mathrm{~m}$ (agl), when it experiences a double engine failure. The only available landing site is the runway that it took off from. The calculations shown in this paper will be used to find if the runway is reachable in two different wind conditions. below

All the aircraft and runway initial conditions are shown

$$
\begin{array}{lll}
\psi_{0}=20^{\circ} & \Gamma=125^{\circ} & F_{x}=-1227 m \\
F_{y}=-9000 & x_{0}=0 & y_{0}=0 \\
V_{w}=30 \mathrm{~m} / \mathrm{s} & \phi=45^{\circ} & h_{\text {init }}=1300 \mathrm{~m}(\mathrm{agl})
\end{array}
$$

As the aircraft was traveling at a high airspeed of 112 $\mathrm{m} / \mathrm{s}$, it is basically unaffected by a light wind, so here a unrealistically high wind speed of $30 \mathrm{~m} / \mathrm{s}$ is used to give a



Figure 8: Four possible maneuvres for the gliding aircraft to the runway in nil wind conditions

$\beta$ of 0.268 to highlight the differences between wind and nil wind conditions.

If the $\psi_{w}$ is set to zero, the four possible trajectories in nil wind can be found, which are shown in Fig. 8. The height lost by the aircraft at the end of the second turn is shown in Table 2. The trajectory chosen is the one which loses least height, which in this case is the path where the initial turn is clockwise and the second turn is anti-clockwise. In the case of no wind the chosen trajectory will be the path which subtends the smallest angle across both turns as it is the shortest, however this may not be the case where wind is present.

TABLE 2: Total height loss over maneuvre for nil wind condition for all four possible trajectories

\begin{tabular}{lll}
\hline$\delta_{1}$ & $\delta_{2}$ & $\Delta h$ \\
\hline-1 & 1 & $1712 \mathrm{~m}$ \\
1 & 1 & $1580 \mathrm{~m}$ \\
1 & -1 & $1030 \mathrm{~m}$ \\
-1 & -1 & $1047 \mathrm{~m}$ \\
\hline
\end{tabular}

In the case where the wind blows at $30 \mathrm{~m} / \mathrm{s}$ from $330^{\circ}$, the four possible trajectories are shown in Fig. 9. The height lost in the glide and the total path distances are shown in Table 3. In this case the optimum trajectory is where both turns are anti-clockwise i.e. $\delta_{1}=-1$ and $\delta_{2}=-1$, as it has the smallest height loss of $964 \mathrm{~m}$. It also has the shortest path length of $12.9 \mathrm{~km}$. However the best path is not necessarily the shortest in wind due to the possibility of higher ground speeds that could be enjoyed on a longer route if a greater portion of that path has a higher tail wind component in the aircraft's direction of travel. An interesting path is $\delta_{1}=1$ and $\delta_{2}=1$, as it subtends an angle greater than $2 \pi$ across the second turn. This has happened as there would not be a smooth path (no tangent) with a turn angle under $2 \pi$ due to the very high wind speeds blowing the aircraft too far downwind, so a point further up wind along the trochoid is used as the start of the turn.

The best $\delta_{1}=-1$ and $\delta_{2}=-1$ trajectory is compared to the best no wind trajectory in Fig. 10. The optimal initial turn for both are different. This is because the initial turn with wind is into wind, as the wind direction blows the 


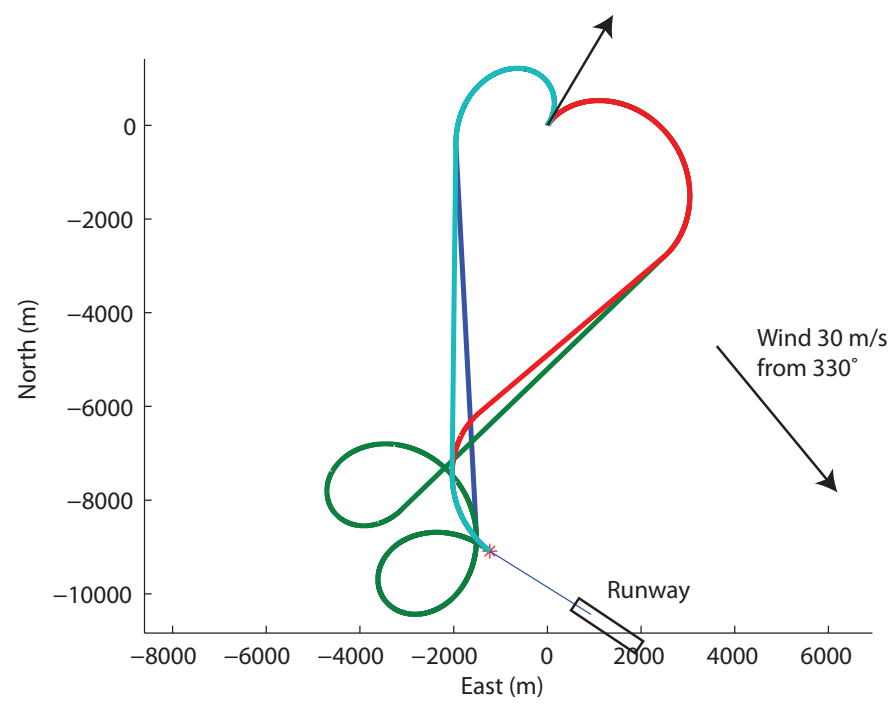

Figure 9: Four possible maneuvres for the gliding aircraft to reach the runway in $150^{\circ} 30 \mathrm{~m} / \mathrm{s}$ wind conditions

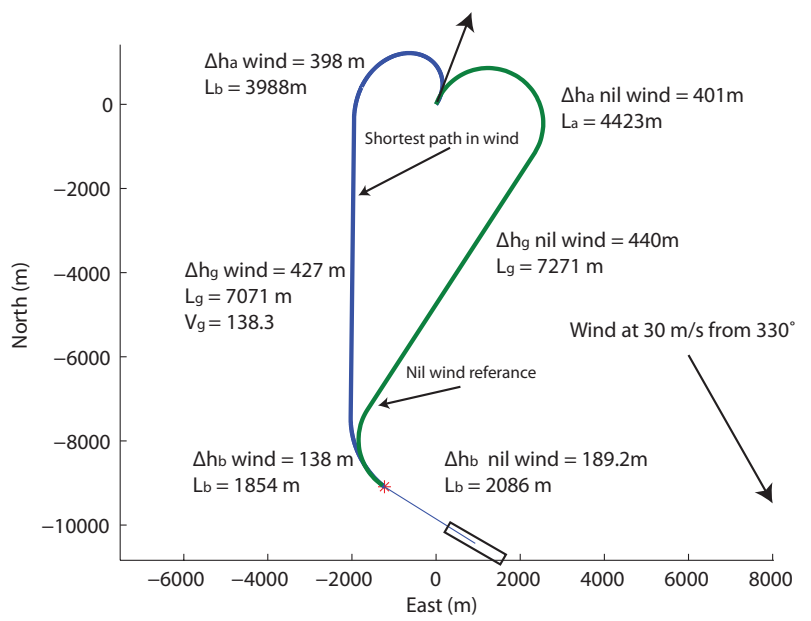

Figure 10: Height profile of aircraft over shortest trajectory for wind from $330^{\circ} 30 \mathrm{~m} / \mathrm{s}$

aircraft towards the landing site this lowers the distance the aircraft has to travel in the level glide. $L_{g}$ in wind is $7071 \mathrm{~m}$ as opposed to $7271 \mathrm{~m}$ in nil wind. It also enables the aircraft to have a greater tail wind component for the level glide with the aircraft's ground velocity at $138 \mathrm{~m} / \mathrm{s}$ whereas in no wind it is traveling at its best glide speed of $V_{i o} 112 \mathrm{~m} / \mathrm{s}$. Both of these together means it loses less height in the level glide. Also as there is an ever increasing tail wind component in the final turn, it loses significantly less height than the nil wind final turn at $138 \mathrm{~m}$ as opposed to $189 \mathrm{~m}$.

Shown in Fig. 11 are the height profiles of the aircraft

TABLE 3: Total height loss and distance travelled for $150^{\circ}$ $30 \mathrm{~m} / \mathrm{s}$ wind for all four possible trajectories

\begin{tabular}{llll}
\hline$\delta_{1}$ & $\delta_{2}$ & $\Delta h$ & Total distance over ground \\
\hline-1 & 1 & $1526 \mathrm{~m}$ & $19.0 \mathrm{~km}$ \\
1 & 1 & $2182 \mathrm{~m}$ & $27.3 \mathrm{~km}$ \\
1 & -1 & $1034 \mathrm{~m}$ & $14.2 \mathrm{~km}$ \\
-1 & -1 & $964 \mathrm{~m}$ & $12.9 \mathrm{~km}$ \\
\hline
\end{tabular}

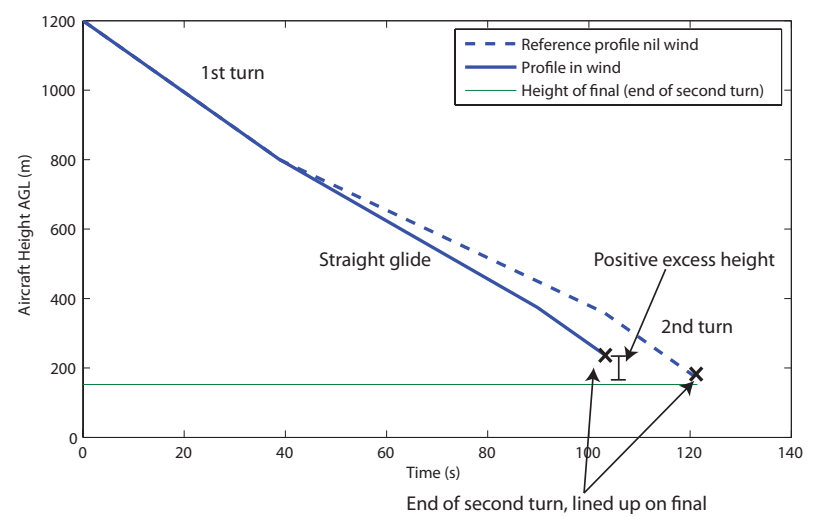

Figure 11: Height profile of aircraft over best trajectory for wind from $330^{\circ} 30 \mathrm{~m} / \mathrm{s}$

over its flight time. It shows the three distinct phases of the flight, and the turns have a steeper height loss as the sink rate is higher in a turn. The site is reachable if by the time it gets to the end of the second turn to line up on final, it is at or above $500 \mathrm{ft}(152 \mathrm{~m})$. It can be seen that for both wind and nil wind both landing sites are reachable since the ends of the profiles are above $152 \mathrm{~m}$. As the wind is in a preferential direction, the trajectory in wind loses less height and thus has more excess height. The excess high in wind is $83.5 \mathrm{~m}$ and $17.2 \mathrm{~m}$, and the excess glide distance is $1380 \mathrm{~m}$ and $284 \mathrm{~m}$ respectively. If a forced landing as needed to be preformed in nil wind, the glide would be extremely marginal.

Paths are generated for the same initial and final conditions but the wind is now from the South, which is much less favorable compared to the last direction. The best path is shown in Fig. 12 which once again compared to the best nil wind path. In this case the best path has the same initial turn direction. This is because the initial direction is at $020^{\circ}$ which is a turn into wind, and it minimises the distance in the first turn because the heading change is smaller.

It can be shown from the height profile in Fig. 13 that the aircraft has a negative excess height of $-47 \mathrm{~m}$, meaning than it does not have adequate height to reach this landing site due to the massively unfavorable wind conditions.

\section{Conclusion}

From a small number of initial conditions and aircraft parameters, a trajectory can be generated to calculate the aircraft minimum height loss to a given landing site in a forced landing situation in the presence of a steady wind. A prediction can be made if the aircraft has enough height to reach a given landing site.

As there are a small number of inputs and even smaller number of outputs, this method is simple and fast enough to be run online. There are big differences in computational time between the same side turn compared to the different side turn. It takes a computer with a Intel i5 $3.33 \mathrm{Ghz}$ processor with $8 \mathrm{~Gb}$ of RAM about $6 \mathrm{~ms}$ for same side turns, this is due to the iterations the algorithm needs to perform changing $h$, and $m$ until the optimum path is found from a number of possibilities. The different side turns it took 15 


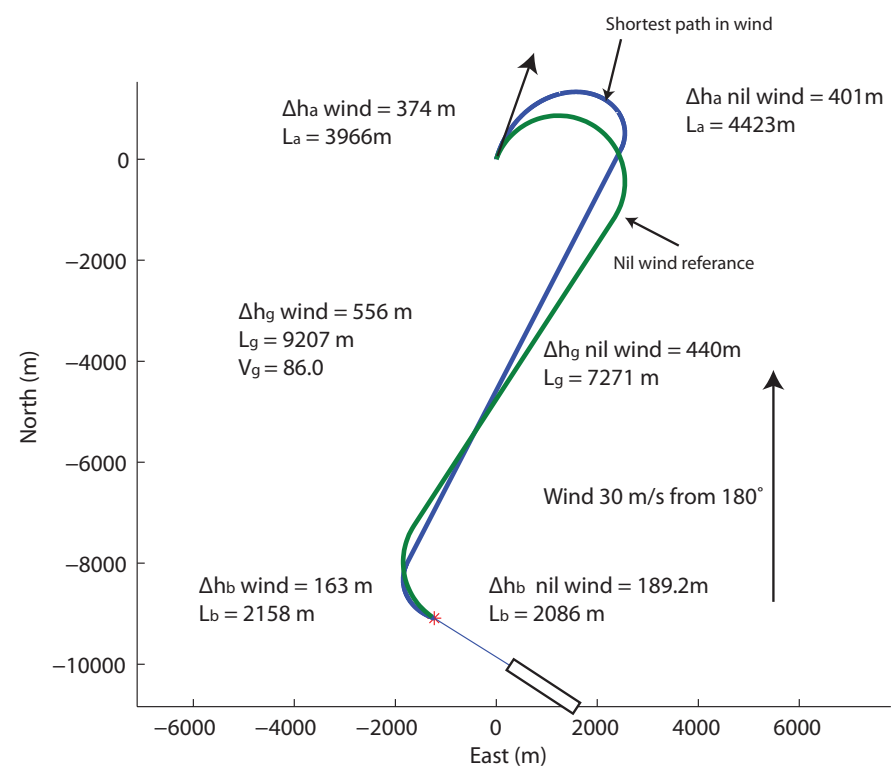

Figure 12: Height profile of aircraft over best trajectory for wind from $180^{\circ} 30 \mathrm{~m} / \mathrm{s}$

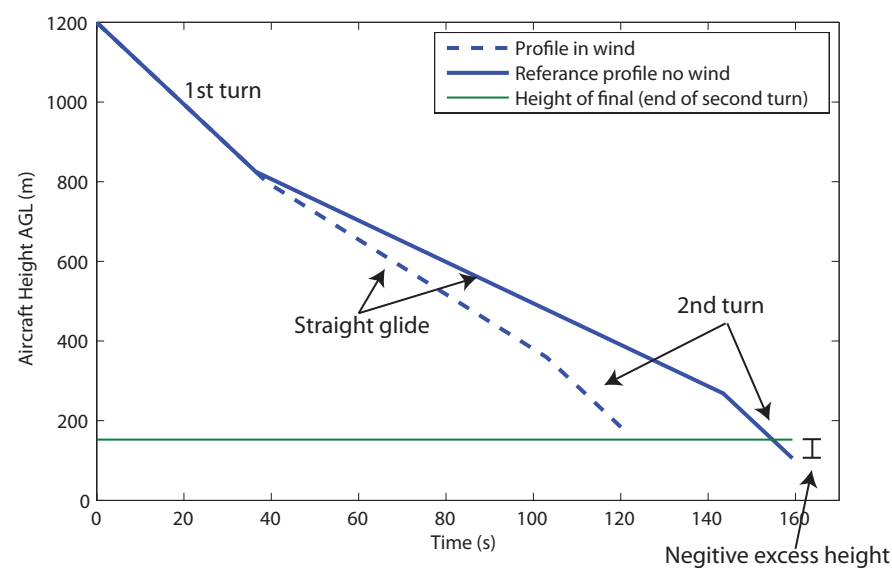

Figure 13: Height profile of aircraft over best trajectory for wind from $180^{\circ} 30 \mathrm{~m} / \mathrm{s}$

ms to run due to the slow running bisection algorithm, but this could be improved by employing a faster root finding technique. Even the slowest of these are more than adequate, as an aircraft will know if it can land at a site in a maximum of $0.0444 \mathrm{~s}$. When the algorithm it run online, this will give an update rate of $22 \mathrm{~Hz}$.

The assumption that the wind is steady is not necessarily true. As an aircraft descends, the wind tends to back as it approaches the surface. How much it back depends on a number of factors, however it does not tend to back more than $30^{\circ}$ so the error introduced is not huge. This work could be extended by adding a term to the base trochoid equation that takes account for a known wind profile. Then the same method could be used to define this slightly different path.

No tangent will be found if the aircraft's engine fails at a distance of less than two turn radius' from the final waypoint $\sqrt{F_{x}^{2}+F_{y}^{2}}<2 R$. However as the aircraft in this case will be very close to the site, it is assumed that it is reachable.

\section{Acknowledgment}

This work was supported by the U.K. Engineering and Physical Sciences Research Council (EPSRC) Autonomous and Intelligent Systems programme under the grant number $\mathrm{EP} / \mathrm{J} 011525 / 1$ with BAE Systems as the leading industrial partner.

\section{References}

[1] NTSB. (2001) 2001 ga accident aircraft data used in annual review. National Transport Saftey Board.

[2] M. A. S. I. A. S. Timothy H. Cox, Christopher J. Nagy, "Civil uav capability assessment," NASA, Tech. Rep., 2004.

[3] M. Coombes, W.-H. Chen, and P. Render, "Reachability analysis of landing sites for forced landing of a uas," Journal of Intelligent \& Robotic Systems, vol. 73, no. 1-4, pp. 635-653, 2014.

[4] D. F. Rogers, "Possible 'impossible' turn," Journal of Aircraft, vol. 32, no. 2, pp. 392-397, 1995

[5] K. Brinkman and H. Visser, "Optimal turn-back manoeuvre after engine failure in a single-engine aircraft during climb-out," Proceedings of the Institution of Mechanical Engineers, Part G: Journal of Aerospace Engineering, vol. 221, no. 1, pp. 17-27, 2007.

[6] D. C. Hyde, "Minimum-altitude-loss gliding turns with terminal constraints(return to runway after engine failure)," in 2005 AIAA Atmospheric Flight Mechanics Conference and Exhibit, 2005, pp. 117.

[7] D. Robson and D. Pooley, Flying Training, 8th ed., A. P. Publishing, Ed. Air Pilot Publishing, 2008.

[8] L. E. Dubins, "On curves of minimal length with a constraint on average curvature, and with prescribed initial and terminal positions and tangents," American Journal of mathematics, pp. 497-516, 1957.

[9] D. Pooley, JAR Flying Training, H. H. Peter Godwin, Daljeet Gill, Ed. Pooley Aviation Ltd., 2003.

[10] L. Techy and C. A. Woolsey, "Minimum-time path planning for unmanned aerial vehicles in steady uniform winds," Journal of guidance control, and dynamics, vol. 32, no. 6, pp. 1736-1746, 2009.

[11] M. Seleck, P. Váňa, M. Rollo, and T. Meiser, "Wind corrections in flight path planning," Int J Adv Robotic Sy, vol. 10, no. 248, 2013.

[12] "The bisection algorithm," in Practical Analysis in One Variable, ser. Undergraduate Texts in Mathematics. Springer New York, 2002, pp. 165-177. [Online]. Available: http://dx.doi.org/10.1007/ 0-387-22644-3_14

[13] M. Abramowitz and I. A. Stegun, Handbook of mathematical functions: with formulas, graphs, and mathematical tables. Courier Dover Publications, 2012.

[14] NTSB, "Loss of thrust in both engines after encountering a flock of birds and subsequent ditching on the hudson river us airways flight 1549 airbus a320-214, n106us weehawken, new jersey january 15, 2009," NTSB, Aircraft Accident Report NTSB/AAR-10/03 PB2010910403, 2010.

[15] M. L. V. Francois DE GERNON and A. RIGALDO, "Performance and design of the airbus a320," Department of Aerospace and Vehicle Engineering Royal Institute of Technology, Tech. Rep., December 2009. 